\title{
Caracterización imagenológica del quiste residual maxilar: Reporte de caso y revisión de la literatura
}

\author{
Imaging characterization of the maxillary residual cyst: Case report and review of the literature \\ Sandro Alexander Lévano Loayza ${ }^{1, a, b}$, Víctor Calderón Ubaquii, ${ }^{2, c, ~ d, ~ A n a ~ P a o l a ~ T r e v e j o-B o c a n e g r a ~}{ }^{2, c, d}$

\section{RESUMEN}

El quiste residual es el resultado de un tejido inflamatorio periapical remanente producto de una extracción dental sin un curetaje minucioso. Mayormente afecta a los varones y a la maxila. Su diagnóstico depende de los hallazgos del examen clínico, radiográfico e histopatológico. El presente caso tiene como objetivo reportar el caso de un quiste residual en la región anterior de la maxila de la cavidad oral de un paciente adulto del Centro Dental Docente Cayetano Heredia con antecedente de extracciones dentales en el área de la lesión hace 10 meses. Se realizó una enucleación y se tomó una muestra para el examen anatomopatológico, el cual posteriormente confirmó el diagnóstico de quiste residual.

PALABRAS CLAVE: Quistes maxilomandibulares, quiste radicular, tomografía computarizada de haz cónico.

\section{SUMMARY}

The residual cyst is a result of remnant periapical inflammatory tissue produced by tooth extraction without a thorough curettage. It mainly affects males and the maxilla. Its diagnosis depends on the clinical, radiographic and histopathological examination findings. The present case aims to report the case of a residual cyst in the anterior region on the maxilla of the oral cavity in an adult patient of the Centro Dental Docente Cayetano Heredia with a history of dental extractions in the lesion area of 10 months ago. An enucleation was performed and a sample was taken for the pathological examination, which subsequently confirmed the diagnosis of residual cyst.

KEY WORDS: Jaw cysts, radicular cyst, cone beam computed tomography.

1 Facultad de Estomatología, Universidad Peruana Cayetano Heredia. Lima, Perú

2 Departamento Académico de Medicina y Cirugía Bucomaxilofacial, Facultad de Estomatología, Universidad Peruana Cayetano Heredia. Lima, Perú

a Cirujano Dentista

Diplomado en Cirugía Oral

Especialista en Radiología Oral y Maxilofacial

Docente 


\section{INTRODUCCIÓN}

Se define al quiste como una cavidad patológica con contenido líquido, semilíquido o gaseoso, revestida interiormente por epitelio y cubierta externamente por tejido conectivo. No es producto de acumulación de pus, suele ser asintomático cuando no hay una infección de por medio y son en su mayoría hallazgos radiográficos. En la región oral y maxilofacial se pueden clasificar en odontogénicos o no odontogénicos (1).

Los quistes odontogénicos de mayor incidencia son los de origen inflamatorio y el Quiste Residual (QR) representa el segundo más frecuente por detrás del quiste apical. Sin embargo, solo representa el $2.8 \%$ de todos los quistes odontogénicos existentes $(2,3)$.

Suele ser asintomático, por lo que frecuentemente es un hallazgo radiográfico, pero cuando alcanza un tamaño considerable puede producir asimetría facial (4).

El crecimiento del QR depende de la capacidad inmunológica del individuo, la virulencia y la proliferación de los restos epiteliales de Malassez en respuesta al proceso inflamatorio crónico que una vez hubo en la región periapical del diente necrótico extraído (5).

El presente caso tiene como objetivo reportar el caso de un quiste residual en la región anterior de la maxila de la cavidad oral de un paciente adulto del Centro Dental Docente Cayetano Heredia con antecedente de extracciones dentales en el área de la lesión hace 10 meses. Se realizó una enucleación y se tomó una muestra para el examen anatomopatológico, el cual posteriormente confirmó el diagnóstico de quiste residual.

\section{REPORTE DEL CASO}

Paciente de sexo masculino de 59 años de edad, acudió al Servicio de Cirugía Oral y Maxilofacial del Centro Dental Docente de la Universidad Peruana Cayetano Heredia, por presentar una lesión nodular en región antero superior en la maxila, con un tiempo de enfermedad de 1 mes, de inicio insidioso y curso progresivo, asintomático. Refirió además que fue sometido a exodoncia simple en la misma región hace 10 meses sin complicaciones aparentes.

No se evidenció ninguna alteración en la evaluación clínica extraoral. Al examen clínico intraoral se confirmó la presencia de la lesión nodular de $1.5 \times 1 \times 1 \mathrm{~cm}$ de diámetro, de consistencia firme, bordes definidos, resilente en la parte central y de coloración violácea. También se evidenció la presencia de un puente protésico de 3 piezas a nivel de $12-21$, y $31-42$ (figura 1). Se le indicó la toma de radiografías panorámica, periapical de zona de pieza 11 y Tomografía Computarizada de Haz Cónico (TCHC) para visualizar la extensión de la lesión y el compromiso de las estructuras anatómicas adyacentes como el conducto nasopalatino, piso de fosas nasales y tablas óseas del maxilar superior.

En la radiografía panorámica se observó una imagen radiolúcida proyectada en el proceso dentoalveolar en zona de pieza 11 alcanzando la cima del reborde alveolar y extendiéndose hasta las superficies radiculares de las piezas 12 y 21 . En la radiografía periapical se comprobó la presencia de la cortical fina en los bordes y se visualizó la continuidad del espacio para el ligamento periodontal de las piezas 12 y 21 (figura 2). En la TCHC, se comprobó la proximidad con el conducto nasopalatino y la expansión, adelgazamiento y erosión de la tabla ósea vestibular (figura 3). Dadas las características imagenológicas las opciones diagnosticas podrían ser: Ameloblastoma Uniquístico, Queratoquiste Odontogénico, Quiste Óseo Simple o Quiste Residual. Unificando los datos de la anamnesis con signos

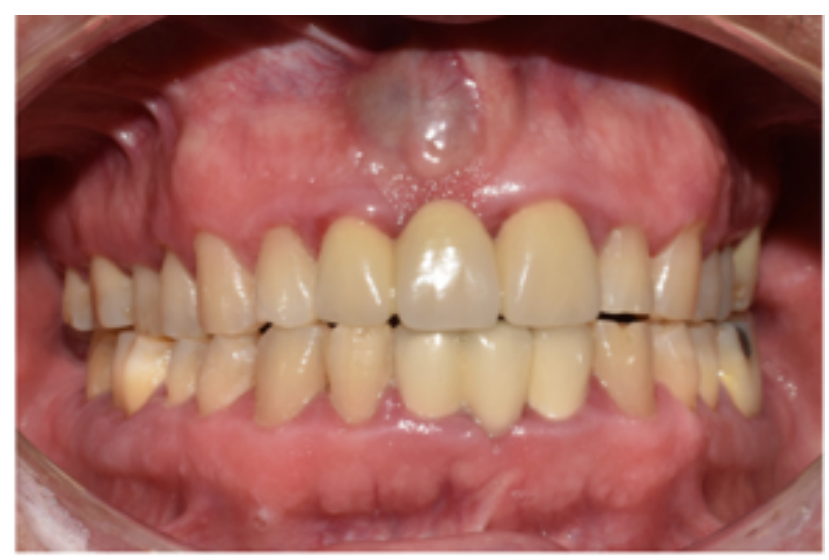

Figura 1. Foto intraoral frontal. Lesión nodular de color violácea de $1.5 \times 1 \times 1 \mathrm{~cm}$ de diámetro 


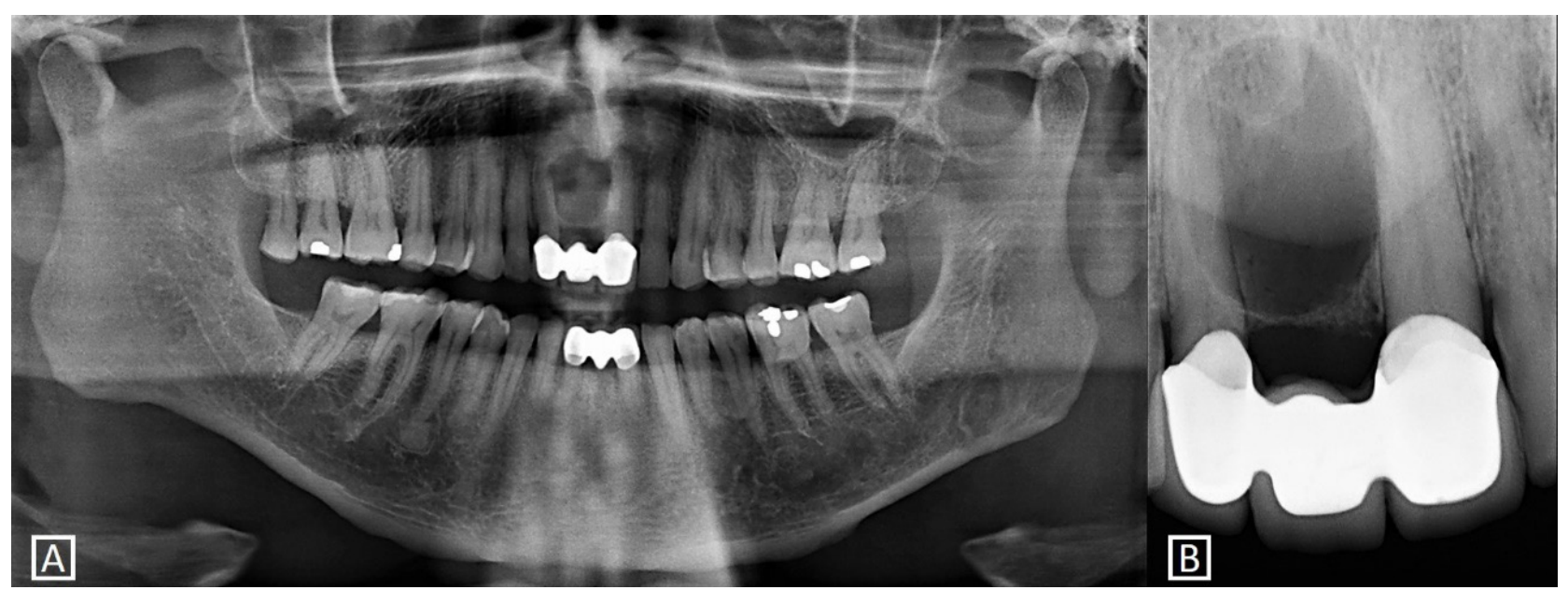

Figura 2. Radiografías A: Panorámica, se observa una imagen radiolúcida redondeada y con bordes corticalizados en zona anterior de maxila. B: Periapical, se evidencia la cortical fina en los bordes y la continuidad del espacio para el ligamento periodontal de las piezas 12 y 21 .
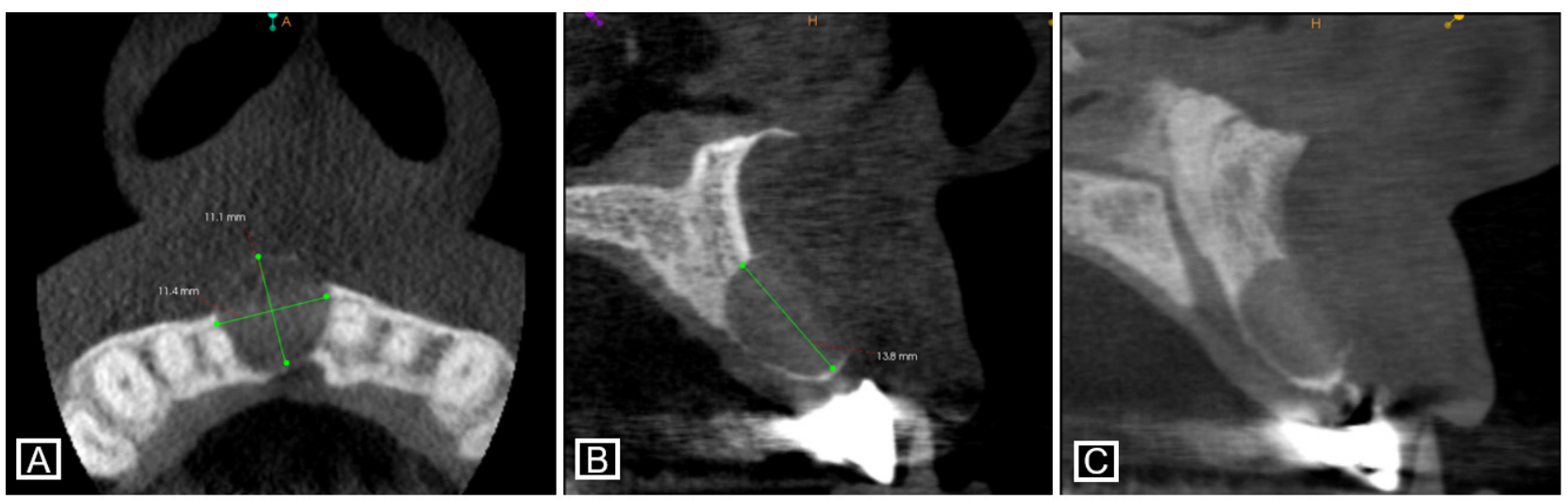

Figura 3. Tomografía Computarizada de Haz Cónico. A: Corte axial, se observa las medidas en sentido antero posterior y latero medial, además se evidencia la expansión, adelgazamiento y erosión de la tabla ósea vestibular y la proximidad con el conducto nasopalatino ocasionando su deformación. B, C: Corte tangencial, se comprueba la erosión de la tabla ósea vestibular y se observa la medida en sentido céfalo caudal. Incidentalmente se observa la morfología poco frecuente del conducto nasopalatino.

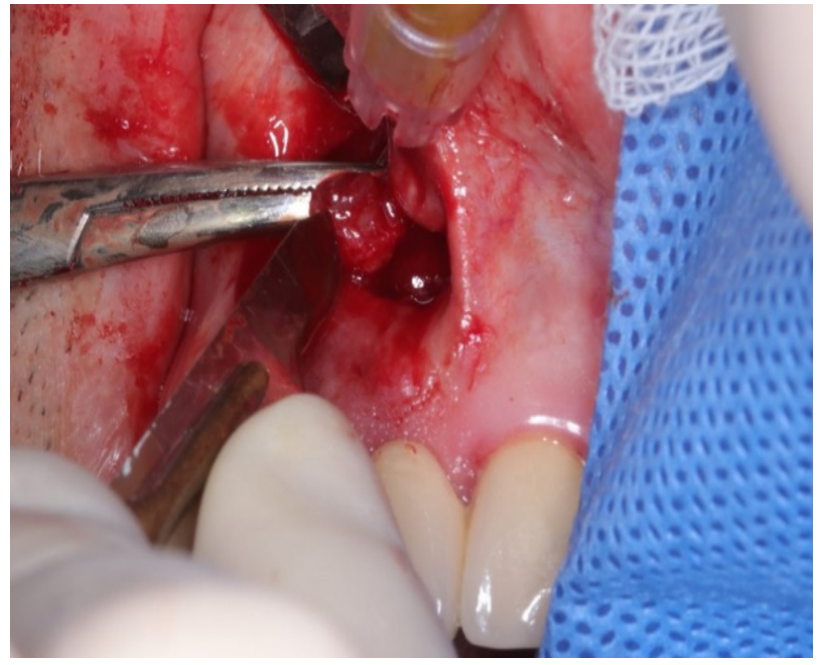

Figura 4. Enucleación de quiste residual clínicos e imagenológicos se concluyó como diagnóstico presuntivo un Quiste Residual.

Se procedió a realizar la enucleación del quiste en la cual se realizó la toma de muestra, osteotomía periférica, curetaje, lavado con $\mathrm{NaCl} 0.9 \%$, sutura y colocación de apósito (figura 4). El resultado del análisis anatomopatológico ratificó el diagnóstico de Quiste Residual.

\section{DISCUSIÓN}

El QR es el resultado de un quiste radicular o granuloma periapical que permaneció en el tejido óseo después de la extracción del órgano dentario asociado a estas patologías (5). 
La frecuencia del QR varía de acuerdo a la población que sea afectada por esta entidad. En estudios previos se han reportado diversas frecuencias del QR que fluctúan entre 1.4\% y 13.7\% (6-11). En Latinoamérica las estadísticas son diferentes. Los estudios realizados en México reportaron 2.3\% (12) y $6.1 \%$ (13), en Chile reportaron $13 \%$ (14) y $0.9 \%$ (15), en Brasil se reportó 4.26\% (16) y en Perú 2.4\% de frecuencia del QR (17).

En el presente reporte se estudia el caso de un paciente varón de 59 años de edad con antecedente de extracción de pieza dentaria en dicha zona. Por lo que las variables demográficas como el sexo y la edad del paciente están dentro de lo informado por otros autores a nivel latinoamericano. Con respecto a la edad existe variación entre los casos reportados a nivel mundial, estos rangos fluctúan entre la tercera y octava década de vida $(6-11,14-16)$ que son edades en las que comienza la pérdida de piezas dentarias por múltiples causas (18). Por otro lado, en los estudios realizados en México y en Perú, son las mujeres las más afectadas con esta lesión $(12,13,17)$.

Radiográficamente se presenta como una imagen radiolúcida bien definida en zona edéntula, con cortical fina o gruesa, que puede estar o no relacionada con el tejido alveolar de un diente extraído (19) o adyacente al reborde alveolar presentando una imagen o patrón en "copa de coñac" esto suele indicar la presencia de una lesión intraósea que se extiende a los tejidos blandos (20). Sin embargo, se han reportado casos de quistes residuales de larga data con presencia de calcificaciones intrínsecas, siendo una característica rara. Ademes suele afectar con mayor frecuencia a la maxila que la mandíbula $(3,19)$. Al tratarse de una imagen única, redondeada u ovalada con presencia de cortical, el QR suele confundirse con un Ameloblastoma Uniquístico, en estadio inicial, Queratoquiste Odontogénico o un Quiste Óseo Simple (21). Cabe recordar que todas estas entidades pueden expandir discretamente las tablas óseas como el QR, pero es la anamnesis la que orienta hacia el diagnóstico correcto (19).

Las técnicas radiográficas convencionales podrían ser idóneas y suficientes para establecer conclusiones imagenológicas de quistes odontogénicos sobre todo cuando se localizan en la premaxila. Sin embargo, la evaluación clínica y la planificación quirúrgica hacen necesaria la evaluación tridimensional de la lesión por la proximidad con estructuras anatómicas de importancia (22). El uso de la TCHC es preferente al de la Tomografía Espiral Multicorte (TEM) a pesar de la posibilidad de la aplicación de sustancias de contraste endovenoso, por la baja dosis de radiación que recibe el paciente, pero sobretodo, por el vóxel isotrópico que posee la TCHC que permite que las mediciones sean idénticas a las que tiene el paciente; sin embargo, la medida del vóxel no es una justificación contundente ya que la TEM puede modificar su vóxel a isotrópico, muchas veces esto no es necesario ya que con su vóxel anisotrópico las diferencias no son estadísticamente significativas. $(19,23,24)$.

La TEM tiene como ventaja sobre la TCHC la medición de las unidades Hounsfield (UH) el cual brinda el valor numérico de la densidad encontrada en el interior de la lesión (19). No obstante, la TCHC poseen herramientas que hacen posible la medición objetiva de la densidad y el cálculo del promedio de gris del vóxel de tejidos como el esmalte dentario, dentina, tejido pulpar, nervio dentario inferior, nervio nasopalatino, entre otros. Estos valores son aproximados y no pueden ser expresados como los UH $(24,25)$. Las medidas obtenidas por los diversos equipos de TCHC no son uniformes. Esta limitación se debe a las deficiencias del panel detector y al efecto del endurecimiento del haz o beam hardening, que hacen posible el fenómeno conocido como cupping por el cual los niveles de gris difieren en el centro comparados con los de la periferia (26).

Se han reportado casos en los que el aumento de la densidad intralesional o incluso la presencia de zonas de calcificación se debe a la cronicidad y a la presencia de sobre infección por otros agentes patógenos $(24,27)$. Dado que se trata de una TCHC no se puede cuantificar este aumento de densidad en UH, además los valores de la escala de grises de un software no se pueden transpolar ni generalizar a otros softwares. Con respecto a la erosión de la tabla ósea vestibular visualizada en el presente caso, se ha encontrado que la mayoría de los quistes odontogénicos en la maxila generan discontinuidad de las tablas óseas (28).

Otro aspecto poco conocido y difundido de los QR es que se han reportado carcinomas odontogénicos que pueden desarrollarse por transformación maligna 
del revestimiento epitelial de quistes odontogénicos. Mosqueda-Taylor et al., hace referencia que según Müllery Waldron mencionan que alrededor del $70 \%$ de estas neoplasias se desarrollan en quistes preexistentes (29). Aunque existen casos originados en la mayoría de las variedades de quistes odontogénicos, el mayor número se ha encontrado asociado a quistes residuales apicales (30).

Histológicamente, un quiste residual comparte características similares con el quiste apical, presentando a menudo un revestimiento epitelial escamoso estratificado, un infiltrado inflamatorio con numerosas hendiduras de colesterol y restos celulares. No obstante, en raras ocasiones se han reportado casos de quistes residuales de larga data con presencia de calcificaciones intrínsecas que son también observadas en radiografías de rutina $(3,19,24,27)$.

Para llegar al diagnóstico definitivo del QR se debe tener en consideración una buena anamnesis y los datos recolectados de los exámenes auxiliares pertinentes, ya que puede confundirse con quistes odontogénicos del desarrollo como el queratoquiste $\mathrm{u}$ otras entidades como el quiste óseo simple y el ameloblastoma unilocular $(6,7,21)$.

Generalmente el tratamiento de elección para el QR es la enucleación quirúrgica, junto con la eliminación del revestimiento del quiste. La tasa de recurrencia es bajo teniendo un buen pronóstico. Sin embargo, es necesario un seguimiento regular para descartar alguna transformación maligna luego de realizado el tratamiento $(3,4)$.

\section{CONCLUSIONES}

Si bien el quiste residual es una lesión ampliamente conocida, es necesario realizar una correcta evaluación clínica, radiológica e histopatológica, para establecer un diagnóstico definitivo certero para realizar un tratamiento adecuado.

En lesiones quísticas siempre es importante la evaluación imagenológica tridimensional con tomografía computarizada para determinar la localización, extensión y compromiso a estructuras adyacentes.
Se debe tener en consideración un curetaje minucioso después de realizar una extracción dentaria de una pieza con patología preexistente, con el fin de evitar un posible desarrollo de un quiste residual en aquella zona.

\section{Correspondencia:}

Sandro Lévano Loayza

Correo electrónico: sandro.levano.1@gmail.com

\section{REFERENCIAS BIBLIOGRÁFICAS}

1. García A, Bujaldón A, Rodríguez A. Lesiones periapicales: diagnóstico y tratamiento. $\mathrm{Av}$ Odontoestomatol. 2015;31(1):31-42.

2. Díaz J, Puig L, Vives C. Perfil epidemiológico, clínico y terapéutico de los quistes odontogénicos en Santiago de Cuba. MEDISAN. 2014; 18(1):52-60.

3. Sridevi K, Nandan SR, Ratnakar P, Srikrishna K, Vamsi Pavani B. Residual cyst associated with calcifications in an elderly patient. J Clin Diagn Res. 2014; 8(2):2469.

4. Rivero Pérez O, Albornoz López del Castillo C, Nápoles González I. Quiste residual gigante: presentación de un caso. Rev Arch Méd Camagüey. 2014;18(5):576-84.

5. Sridevi K, Nandan R, Ratnakar P, Srikrishna K, VamsiPavani B. Residual cyst associated with calcifications in an elderly patient. J Clin Diagn Res. 2014; 8(2): 2469.

6. Bajaj N, Gala V, Tirpude V, Belur A, Fernandes G. A Retrospective Cone Beam Computed Tomography (CBCT) Study of the Prevalence of Incidental Odontogenic Cyst Findings in an Indian Subpopulation. IJSRST. 2018; 4(2): 800-6.

7. Khosravi N, Razavi SM, Kowkabi M, Navabi AA. Demographic distribution of odontogenic cysts in Isfahan (Iran) over a 23-year period (1988-2010). Dent Res J. 2013; 10(2):162-7.

8. Acikgoz A, Uzun-Bulut E, Ozden B, Gunduz K. Prevalence and distribution of odontogenic and nonodontogenic cysts in a Turkish Population. Med Oral Patol Oral Cir Bucal. 2012;17(1):e108-e15

9. Baghaei F, Zargaran M, Najmi HR, Moghimbeigi A. A Clinicopathological Study of Odontogenic Cysts and Tumors in Hamadan, Iran. J Dent Shiraz Univ Med Sci. 2014; 15(4): 167-72.

10. Nunez-Urrutia S, Figueiredo R, Gay Escoda C. Retrospective clinicopathological study of 418 odontogenic cysts. Med Oral Patol Oral Cir Bucal. 2010;15(6):e767-e73.

11. Kambalimath DH, Kambalimath HV, Agraval SM, Singh M, Jain N, Anurag B, et al. Prevalence and 
Distribution of Odontogenic Cyst in Indian Population: A 10 Year Retrospective Study. J Maxillofac Oral Surg. 2014; 13(1):10.

12. Villasis-Sarmiento L, Portilla-Robertson J, MelendezOcampo A, Gaitan-Cepeda LA, Leyva-Huerta ER. Prevalence and distribution of odontogenic cysts in a Mexican sample. A 753 cases study. J Clin Exp Dent. 2017; 9(4):e531-8.

13. Ledesma-Montes C, Hernandez-Guerrero JC, GarcesOrtiz M. Clinico-pathologic study of odontogenic cysts in a Mexican sample population. Arch Med Res. 2000;31(4):373-6

14. Ochsenius G, Escobar E, Godoy L, Penafiel S. Odontogenic cysts: analysis of 2,944 cases in Chile. Med Oral Pathol Cir Bucal. 2007;12(2): E85-E91.

15. Peters F I, López G P, Preisler E G, Sotomayor C C, Donoso Z M, Hernández V S. Prevalencia de quistes odontogénicos, Hospital Regional Valdivia entre los años 1990 y 2010. Av Odontoest. 2012; 28 (6): 303-9.

16. Prockt AP, Schebela CR, Maito FDM, Sant ana-Filho M, Rados PV. Odontogenic cysts: analysis of 680 cases in Brazil. Head and Neck Pathol. 2008; 2:150-6.

17. Moya D. Prevalencia de Quistes Odontogénicos en el Hospital Nacional Hipólito Unanue en el periodo 20042009. Tesis de Título Profesional. Lima, Perú. Universidad Nacional Mayor de San Marcos. 2011.

18. Bitencourt FV, Corrêa HW, Toassi RFC. Tooth loss experiences in adult and elderly users of Primary Health Care. Cien Saude Colet. 2019; 24(1):169-180.

19. White SC, Pharoah MJ. Oral Radiology principles and interpretation. 5ta ed. St Louis: Mosby; 2006.p. 59798.

20. Bello C. Diagnósticos diferenciales de las lesiones radiolúcidas de los maxilares. Tesis de Especialista. Caracas, Venezuela. Universidad Central de Venezuela. 2002.

21. Gham A, Mhaske Sh. Cyst of the orofacial region. En: Textbook of Oral Pathology. 2da ed. Nueva Delhi: Jaypee publishers; 2010.p.364.

22. Boopathi D, Vannathan J, Subramanian S, Mariappan J. Conventional radiograph and cone-beam computed tomography in the evaluation of odontogenic cysts and tumors - An analysis of seven cases. J Res Dent Sci. 2020; 54-9.
23. Panjnoush M, Norouzi H, Kheirandish Y, Reza A, Mofidi N, Evaluation of Morphology and Anatomical Measurement of Nasopalatine Canal Using Cone Beam Computed Tomography. J Dent. 2016;13(4): 287-94.

24. Mishra SS, Degwekar SS, Banode PJ, Bhowate RR, Mukta BM, Mishra PS. Comparative study of cone beam computed tomography and multislice computed tomography in the radiographic evaluation of cyst and tumors of the jaw. J Indian Acad Oral Med Radiol. 2014;26: 2539.

25. Nasim A, Sasankoti Mohan RP, Nagaraju K, Malik SS, Goel S, Gupta S. Application of cone beam computed tomography gray scale values in the diagnosis of cysts and tumors. J Indian Acad Oral Med Radiol. 2018; 30:4-9.

26. Reeves TE, Mah P, McDavid WD. Deriving Hounsfield units using grey levels in cone beam CT: a clinical application. Dentomaxillofac Radiology. 2012; 41: 500-8.

27. Shibasaki M, Iwai T, Chikuma H, Mitsudo K, Inayama Y, Tohnai I. Actinomyces associated calcifications in a dentigerous cyst of the mandible. J Craniofac Surg. 2013;24(3):311-4.

28. Yoshiura K, Higuchi Y, Araki K, Shinohara M, Kawasu T, Yuasa K, et al. Morphologic analysis of odontogenic cysts with computed tomography. Oral Surg Oral Med Oral Pathol Oral Radiol Endod. 1997; 83(6):712-8.

29. Mosqueda-Taylor A, Meneses A, Godoy L, Suárez M, Luna K. Tumores odontogénicos malignos. Estudio retrospectivo y colaborativo de 7 casos. Med Oral. 2003; 8:110-21.

30. Morales D, Sánchez JG, Camacho CG. Carcinoma primario intraóseo mandibular derivado de un quiste odontogénico. Rev Cubana Estomatol. 2018; 55(1):7381.

Recibido : 16-08-2020

Aceptado : 16-12-2020 